\title{
A comparative study of the thermal behavior of three different 3D printer liquefiers
}

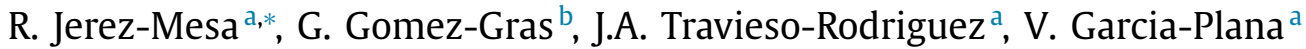 \\ ${ }^{a}$ Mechanical Engineering Department, Escola d'Enginyeria de Barcelona Est, Universitat Politècnica de Catalunya, Edifici A. Campus Diagonal-Besòs. Av. \\ d'Eduard Maristany, 10-14, 08019, Barcelona, Spain \\ ${ }^{\mathrm{b}}$ Industrial Engineering Department, Institut Químic de Sarrià, Universitat Ramon Llull, Via Augusta, 390, 08017, Barcelona, Spain
}

\section{A R T I C L E I N F O}

\section{Article history:}

Received 5 December 2016

Revised 16 May 2017

Accepted 15 June 2017

Available online $\mathrm{xxx}$

\section{Keywords:}

3D printing

RepRap

Fused deposition modeling

Liquefier

FEM

\begin{abstract}
A B S T R A C T
The expansion of 3D printing systems as mechatronic devices able to localize manufacturing activities has attracted the attention in academic and professional labs worldwide. However, little is known from a formal point of view about the actual possibilities of optimization in terms of hardware and software. This paper proposes alternative geometrical designs of RepRap 3D printer liquefiers, and offers an evaluation of their thermal performance by analyzing the temperature profiles derived from their functioning in a steady state regime. For that purpose, finite element calculation and experimental techniques are combined and compared. A detachable model of liquefier, used in numerous RepRap 3D printers, has been taken as a reference to design and manufacture two new variations. As the objective of the liquefier is to heat the plastic material while limiting the temperature at its top, heat conduction must be prevented by means of a refrigerating fan faced at the liquefier body. The effect of that fan has been introduced in the system of study, by evaluating the influence of five different fan velocities on the final temperature profile. All combinations of fan speed and liquefier geometry have been calculated through a finite elements model. Then, they have been reproduced experimentally, measuring discrete temperatures at different heights of the liquefier by a group of thermocouples installed on the surface of the fins composing the liquefier heat sink. Results showed that the defined FEM model reproduces acceptably the final temperature profiles obtained by experimental measures. Furthermore, the new proposed design provided with thick fins shows its good performance when the 3D printing process is performed with a fan speed higher than $10 \%$ (defined according to a PWM function programmed at the 3D printer's firmware), while its design presents a much lower manufacturing time with regards to the present available design. It is also concluded that refrigerating the liquefier during a standard 3D printing process with an airflow higher than $\mathrm{PWM}=20 \%$ is not recommended, due to a fall in efficiency and not remarkable refrigerating effect obtained through it.
\end{abstract}

(c) 2017 Published by Elsevier Ltd.

\section{Introduction}

Additive manufacturing systems have been used for commercial purposes and proficient prototyping and manufacturing since the 70s. Stereolitography, metal laser sintering, laminated object manufacturing or fused deposition modeling (FDM) are some of the most extended additive manufacturing technologies [1]. Small scale 3D printing devices, which can nowadays often be found in local production labs have been the result of the evolution of existing industrial technologies. The promoters of that scale reduction and development was a group of students from the University of

\footnotetext{
This paper was recommended for publication by Associate Editor Dr Kira Barton.

* Corresponding author.

E-mail address: ramon.jerez@upc.edu (R. Jerez-Mesa).
}

Bath lead by Professor Adrian. They succeeded in conceiving and building a self-reproducible open source 3D printer, in the framework of a project called the Replicating Rapid Prototyper initiative, also known as RepRap [2].

Additive or layer by layer manufacturing consists in obtaining a desired geometry by adding progressively a raw material into the manufacturing environment. That operation can be performed through different technologies, so that each one derives in a particular 3D printing device. The technology on which most open source 3D printers are based is fused deposition modeling (FDM) (Fig. 1). The raw material is available as a filament of extruded material, and is introduced into the system by a mechanical feed mechanism, for instance a pair of knurled rollers. The material is led into a liquefier body along which the filament is progressively heated up, until the glass transition temperature of the material 


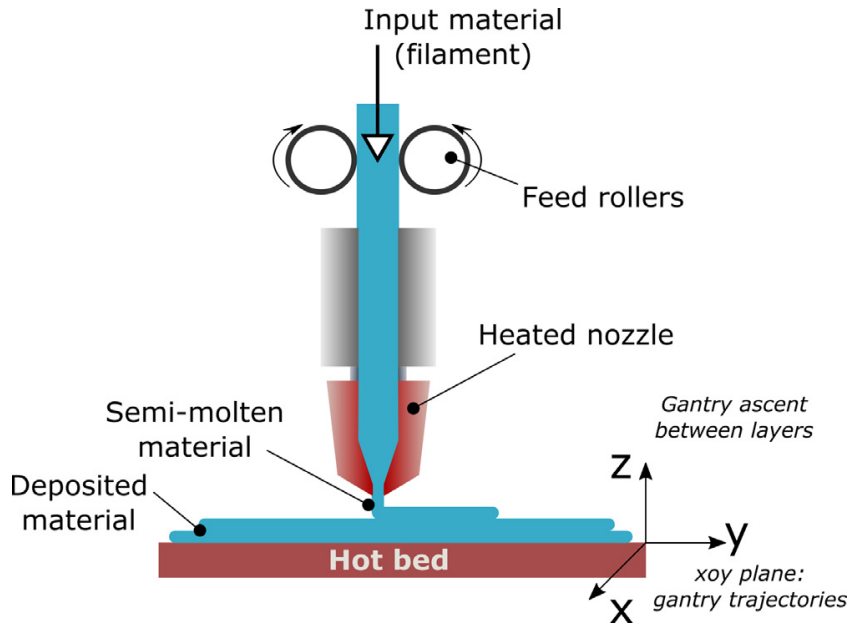

Fig. 1. Schematic representation of a fused deposition modelling system.

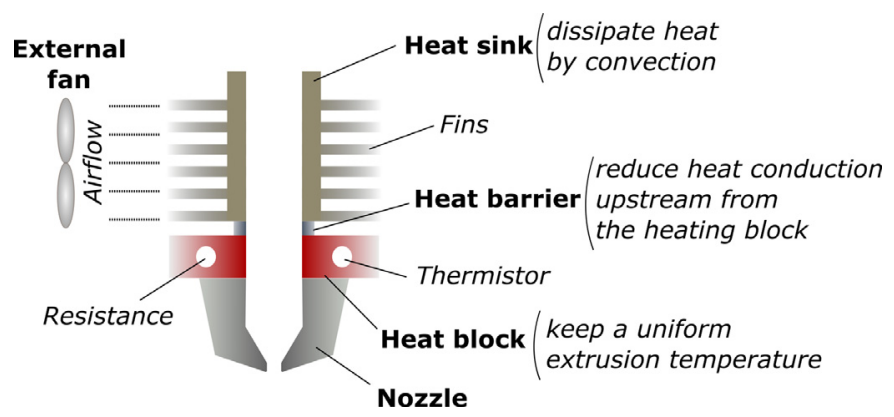

Fig. 2. Structure and parts of a standard FDM liquefier used in this study.

is surpassed [3]. Its subsequent change of state into a thick semimolten material, allows it to be extruded through a nozzle at the end of the liquefier. The tip of the nozzle has a controlled diameter, whose value influences the quality of the printed part and the productivity of the process [4]. Raw materials are presently delivered in $1.75-\mathrm{mm}$ and $3-\mathrm{mm}$ diameter filaments, and nozzle diameters can vary from 0.1 to $1 \mathrm{~mm}$, so that the maximum diameter constriction achievable by desktop FDM 3D printers can ascend to a proportion of 1:30. The described extrusion system is mounted on a motorized gantry, which deposits the material continuously onto the horizontal plane, in subsequent steps or layers separated a certain dimension called layer height.

During the printing process, two components of the printing device must be kept at a certain temperature. Firstly, the hot bed, that is, the platform on which the first layer of material is laid, and which is therefore the base of the manufactured part. The bed temperature is usually kept inside the $50^{\circ} \mathrm{C}$ to $80^{\circ} \mathrm{C}$ fork, to ensure the steady adhesion of the first layer for a proper building of the rest of the part. The liquefier is the second part subjected to temperature control, as it is the responsible for the hot extrusion process of the filament.

Conventional liquefiers can be divided in four main components, as shown in Fig. 2. The nozzle is responsible for the diameter constriction of the filament, and its temperature must be kept constant to ensure a correct material flow. That temperature is achieved by heat inflow from a neighboring a resistance, embedded inside the heating block to which the nozzle is attached. A second body is housed inside the heating block, that is, a thermistor sensor to monitor the temperature. Upstream of the heating block, all the liquefier components are destined to reduce heat flow towards the opposite end of the liquefier body. A low section heat barrier made from a low conductive stainless steel, joins the heat block with the rest of the liquefier body, and prevents heat flow.
The liquefier body is composed of a finned surface refrigerated by an external fan.

Minimizing that heat flow upwards is necessary to save energy during the printing process, as the more heat is lost from the heat block, the longer must the resistance work to keep at an appropriate level the objective nozzle temperature. Secondly, if that temperature fluctuates too much, it could eventually lead the nozzle channel to obstruction and discontinuous flow, with the consequent loss of final quality of the part [5]. Hardware maintenance and durability reasons also justify the need to prevent upstream heat flow. As 3D printed parts are used to fix the liquefier to the printing gantry, prolonged overtemperature at the top of the liquefier could lead those parts to failure or irreversible deformation making them non-functional. In effect, PLA, a material typically used in FDM 3D printers, starts to experience deformation at $35^{\circ} \mathrm{C}$ [6].

The great importance of temperature control during the 3D printing process can be noticed by consulting the available bibliography. Most researchers tackle with the thermal analysis of 3D printing as a process factor influencing the manufactured part. With that aim, they analyze the direct effect on bonding strength between filaments, and relate them to specific mechanical behavior descriptors. For instance, Bellehumeur et al. [7] observed that the cohesion between layers can be enhanced by ensuring the adequate thermal behavior of the system, so that the freshly extruded material is able to combine chemically to the already deposited material by effect of neck growth between filaments and diffusion of polymeric chains. Bellini et al. [8], investigated flexural and tensile stress of ABS specimens, and found that the elastic modulus and critical strength values in both cases were the lowest for specimens built in the xoz plane. This was explained because they show the weakest bonding strength of all tested orientations, and therefore, the thermal behavior of the liquefier has strong repercussions on the mesostructured of 3D printed parts. Similar assessments can be found for PLA [9] and polycarbonate specimens [10]. Other authors show the correlation of thermal behavior on the residual stress of 3D printed parts [11-13], but in no case the evolution of temperatures or the heat dissipation mechanisms during the $3 \mathrm{D}$ printing process are considered.

Albeit the proved extensive bibliography studying the influence of temperature during a $3 \mathrm{D}$ printing process, the behavior of $3 \mathrm{D}$ printer liquefiers as a function of their geometry and interaction between parts has not been studied whatsoever. Classical papers refer to the thermal behavior of industrial extruders. For instance, Huang and Peng [14] developed a mathematical model of the dispersive melting process of plastics in industrial screw extruders. However, this design is far from the actual construction of a 3D printer extruder. Yardimci [15] found that the bonding quality of filaments was highly dependent on the heat transfer mechanisms. Later, the same author quantified the influence of the nozzle design on the thermal behavior of 3D printers through the Biot and Peklet adimensional numbers, through an analytical model [16]. Bellini et al. [17] developed a mathematical model through a transfer function as a means of achieving a more accurate understanding of the thermal interaction between the liquefier and the printing material. Bellehumeur et al. [18] highlighted in their conclusions that the control of the extruding temperature is fundamental to guarantee a proper neck growth between deposited filaments. The literature also provides reference to FEM models of FDM liquefiers, focusing on the thermal behaviour of specific materials due to their thermal interaction with the liquefier body [19-22].

The previous paragraphs present previous research items related to the aim of this paper. These conclusions evidence the importance of temperature control during 3D printing due to heat transfer mechanisms. However, the models and analytical expressions developed in those references were not applied to actual liq- 


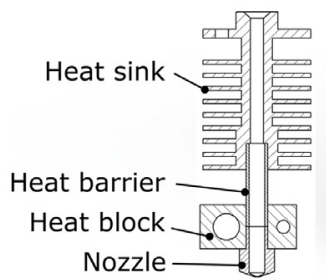

(a)

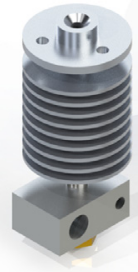

(b)

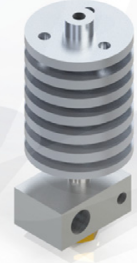

(c)

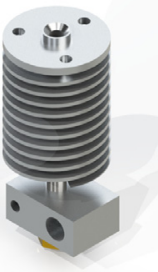

(d)
Fig. 3. Liquefier geometries considered in the study. (a) Cross section of BCNozzle model liquefier. (b) Original design: BCNozzle liquefier. (c) Thick-finned Twist3D liquefier. (d) Helicoidal-shaped Twist3D liquefier.

uefier designs, which is the objective of this work. No comparative approach between different feasible solutions was considered either. This paper starts from previous works presented in JerezMesa et al. [23], where a finite elements model was developed to simulate the thermal behavior of a commercial 3D printer liquefier. The model is here used to compare three different liquefier solutions. As convective dissipation mechanisms play an important role in the model, six different air inflows shall be tested. Then, the obtained temperature profiles will be compared with experimental data taken with a thermocouple setup on the real manufactured liquefiers, to evaluate which printing configuration allows to save more energy during the printing process.

Succeeding in modeling 3D printer liquefiers is a necessary milestone to acquire a wider knowledge of the process dynamics, thus being able to design cheaper, and more durable and precise 3D printing devices. The availability of improved finite element models is a critical tool to understand the heat dissipation effectiveness of a certain liquefier, and leads directly to the design of optimized geometries. It also allows to control critical aspects of the process, such as the localization of the melting front, very relevant to prevent the liquefier from failing in feeding the system with the input material due to eventual blocking and other phenomena associated with an incorrect thermal performance of hardware components.

\section{Materials and methods}

The BCNozzle liquefier, designed at the BCN3D Technologies Foundation, has been taken as the basis for the design and manufacturing of the other two liquefiers object of study. Fig. 3a shows the section of this liquefier, and the parts of which it is composed, already introduced in the previous section at Fig. 2. The thermal block and heat sink are built of AW-3033-H18 aluminum, the nozzle tip is EN CW614N brass, and the heat barrier is manufactured of 1.4306 stainless steel.

\subsection{Liquefier designs}

Fig. 3b-d show rendered images of the three liquefiers, showing the geometrical differences among them, which lies in the different geometry of the fins along the heat sink. The fins of the BCNozzle liquefier are shaped as parallel 1-mm thick cylinders. The second liquefier included in this study, named X-Truder, is a direct variation of this one. The fins along its surface have been thickened up to $4 \mathrm{~mm}$. This solution was inspired on theoretical results related to forced convective heat dissipation of finned cylinders, which showed that the increase of fin thickness derives in higher Nusselt $(\mathrm{Nu})$ adimensional number (Eq. (1)).

$N u=\frac{h \cdot L}{k}$
Table 1

Tested conditions for temperature monitoring during the experimental essays and for FEM analysis.

\begin{tabular}{ll}
\hline Factor & Levels \\
\hline Fan velocity (PWM function) & $10 \%-12 \%-15 \%-20 \%-30 \%$ \\
BCNozzle & BCNozzle - Twist3D - Xtruder \\
\hline
\end{tabular}

where $h$ is the convection heat transfer, $L$ is the fin characteristic length, and $k$ is the heat sink conductivity.

As $\mathrm{Nu}$ represents the ratio of convective transfer to conductive heat conduction, a higher $\mathrm{Nu}$ value indicates higher convective dissipation. According to Bassam [24], fin thickness is the most influential parameters on the $\mathrm{Nu}$ number, and the new 4-mm thickness fins will give way to higher values. Therefore, heat dissipation is expected to rise with positive effects on the liquefier thermal performance.

The second alternative geometry of the dissipation fins is called Twist3D liquefier. This design is based on a heat sink geometry shaped as a continuous helicoidal fin around the central channel. This geometry allows to increase the contact time between the cooling air and the heat sink surface, thus improving heat conduction by convection between both of them. The positive effects of this intended increase of turbulence induced by a helicoidal channel is to be confirmed experimentally in this work. Although the manufacturing time of this heat sink would be higher due to its more complex shape, if results were considerably better, it could be a feasible solution for future designs.

Once both new liquefiers have been designed and manufactured at the university workshop with a CNC lathe, they can be easily mounted on the system, as they are screwed to the heat barrier to ensure interchangeability.

\subsection{Simulated and tested $3 D$ printing conditions}

The temperature profile of the three described liquefiers is studied for five different fan velocities: $10 \%, 12 \%, 15 \%, 20 \%$ and $30 \%$, and the unventilated case (Table 1). Their definition in terms of percentages is based on the PWM (pulse width modulation) output signal which electronically controls the fan velocity at the 3D printer's Arduino motherboard. That percentage value is the ratio of the average voltage feeding the fan with regards to the maximum average voltage admissible by the device, so that when that voltage is lowered, the fan is turned on at a lower speed. This is the way that most 3D printing software dashboards are designed to control the fan speed applied during the additive process. Although it is an easy way to regulate the convective air inflow, it is not precise, as the user cannot decide specifically what volume of air to propel per time unit. A correspondence between the proportional value established by the PWM signal regulation through a slide bar in the 3D printing software, and the actual air propelled by the San Ace40 Sanyo Denki fan is found in Nikzad et al. [20].

The combination of all airflow levels with the three geometries gives way to 15 different testing conditions, all of them calculated with a FEM, and measured in situ through the experimental setup explained below. In the last case, a reference curve measured in the case of no convective dissipation mechanism is included. The following paragraphs explain the designed FEM and the experimental setup used to take the in situ measurements.

\subsection{Description of the finite elements model}

The FEM used for this study is the one described by Jerez-Mesa et al. [23], performed with the COMSOL Multiphysics 4.4 software. The model works by solving the continuity equation (Eq. (2)), the 
Navier-Stokes equation for Newtonian fluids (Eq. (3)), and the energy equation (Eq. (4)) [25,26], all three of them in the stationary state, that is:

$\nabla \cdot(\rho \mathbf{u})=0$

where $\rho$ is the fluid density and $\mathbf{u}$ is the fluid velocity.

$\rho(\mathbf{u} \cdot \nabla \mathbf{u})=-\nabla p+\nabla \cdot\left(\mu\left(\nabla \mathbf{u}+(\nabla \mathbf{u})^{\mathrm{T}}\right)-\frac{2}{3} \mu(\nabla \cdot \mathbf{u}) \mathbf{I}\right)+\mathbf{F}$

where $p$ is the fluid pressure, $\mu$ is the fluid dynamic viscosity, $\mathbf{I}$ is the identity matrix, $\mathrm{T}$ is the temperature and $\mathbf{F}$ are the external forces applied to the fluid.

$\rho C_{p} \mathbf{u} \cdot \nabla T=\nabla \cdot(k \nabla T)+\dot{Q}$

where, $C_{p}$ is the calorific capacity at constant pressure, $k$ is the thermal conductivity, and $\dot{Q}$ is the heat flow.

The model was run for a mesh composed of 171,517 elements, and an approximate computing time of $10 \mathrm{~min}$ for each tested condition. Once the model was calculated, the resulting temperature has been acquired with the graphic interface of the software, taking the isothermal map at the symmetry plane of the heat sink, and at four points at $0,15,22$ and $30 \mathrm{~mm}$ heights, considering the lowest point of the heat sink as reference.

\subsection{Experimental setup}

To simplify the experimental setup, the two main components - fan and liquefier - were attached to a PLA supporting structure, designed and manufactured with a 3D printer specifically for this testing application (Fig. 4a). The dimensions of the structure allowed to face the fan and the heat sink of each tested liquefier at the same height and distance $(20 \mathrm{~mm})$ during the whole measuring process, emulating the actual arrangement of both components in a real 3D printer. The aim of using this structure, was to simplify the handling of the different sensors, especially the installation of the four SE000 K-type thermocouples inside the liquefier fins every

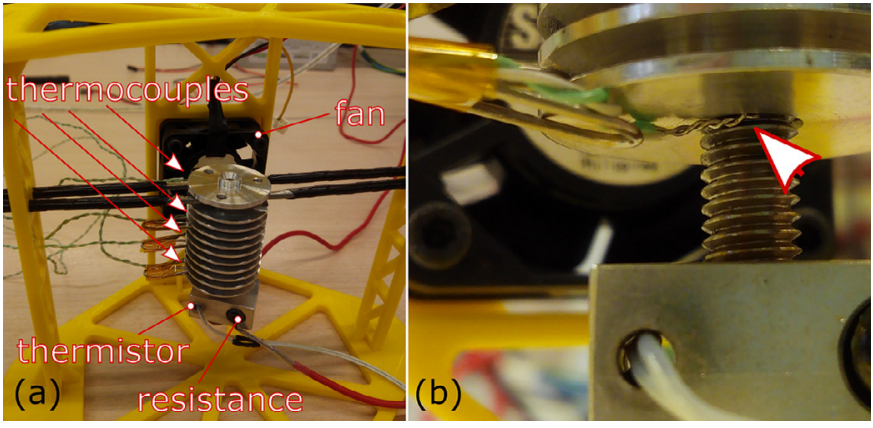

Fig. 4. (a) Fan and liquefier mounted on the PLA support structure. The four thermocouples are fixed at different levels of the liquefier body (b) Detail of liquefier fixation and position of the lowest thermocouple.

time the liquefier body was changed (Fig. 4b). These thermocouples are formed by a chrome-constantan twisted pair conductor, its conductor finished as exposed flat tips. They were specifically fixed with Kapton tape to the fins correspondent to the 0, 15, 22 and $30-\mathrm{mm}$ heights.

Once the fan and liquefier were attached, and the thermocouples installed, the resistance and thermistor were hosed inside the heating block (Fig. 4a). Then, both thermal components and the refrigerating fan were connected to a RepRap 3D printer Arduino motherboard, governed by the open source Marlin firmware for these kind of devices (Fig. 5). The Arduino motherboard was then connected through USB port to a computer, so that fan speed and objective temperature could be adjusted manually through the RepetierHost freeware. In effect, the Marlin firmware changes the on/off state of the resistance in order to keep the extruding temperature at a desired objective, according to the information received by the thermistor, which continuously monitors the temperature achieved at the heat block. The temperature adjustment works, therefore, as a simple feedback loop.

The measuring protocol can be described as follows. Once the whole setup was arranged, the resistance was turned on, establish-

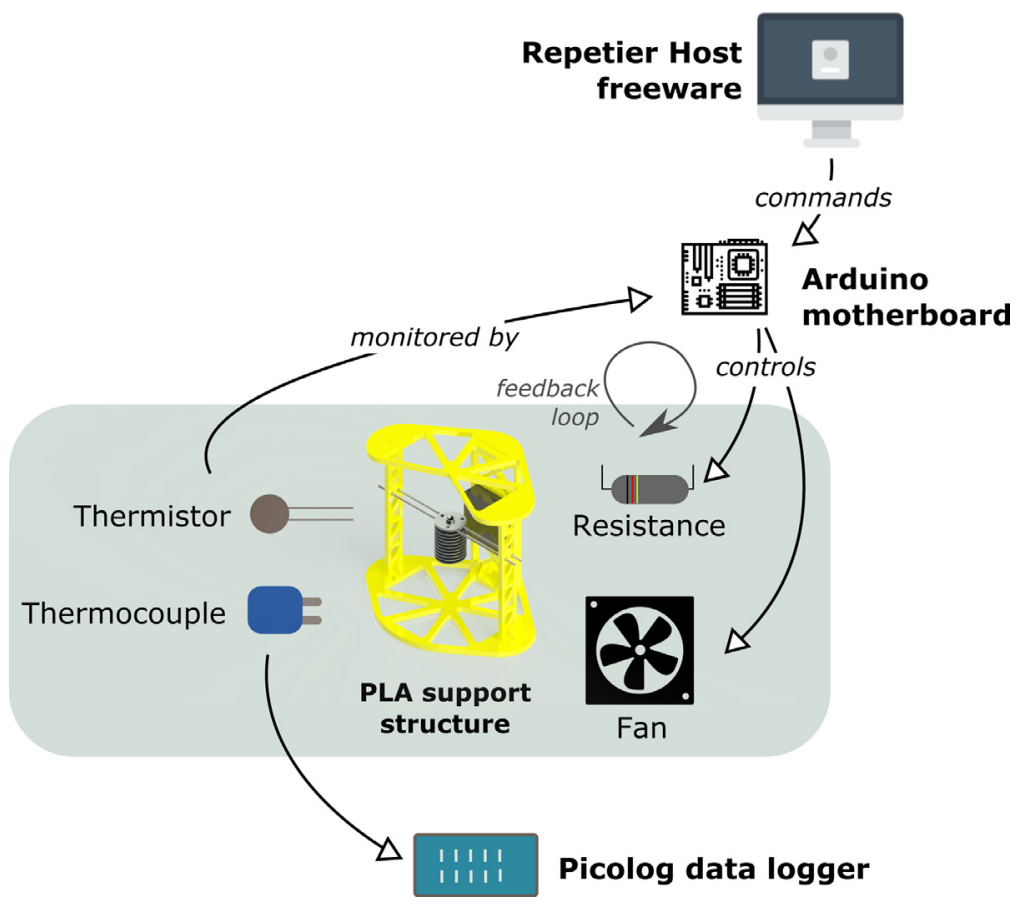

Fig. 5. Representation of the components used for the experimental measurement of temperatures. 

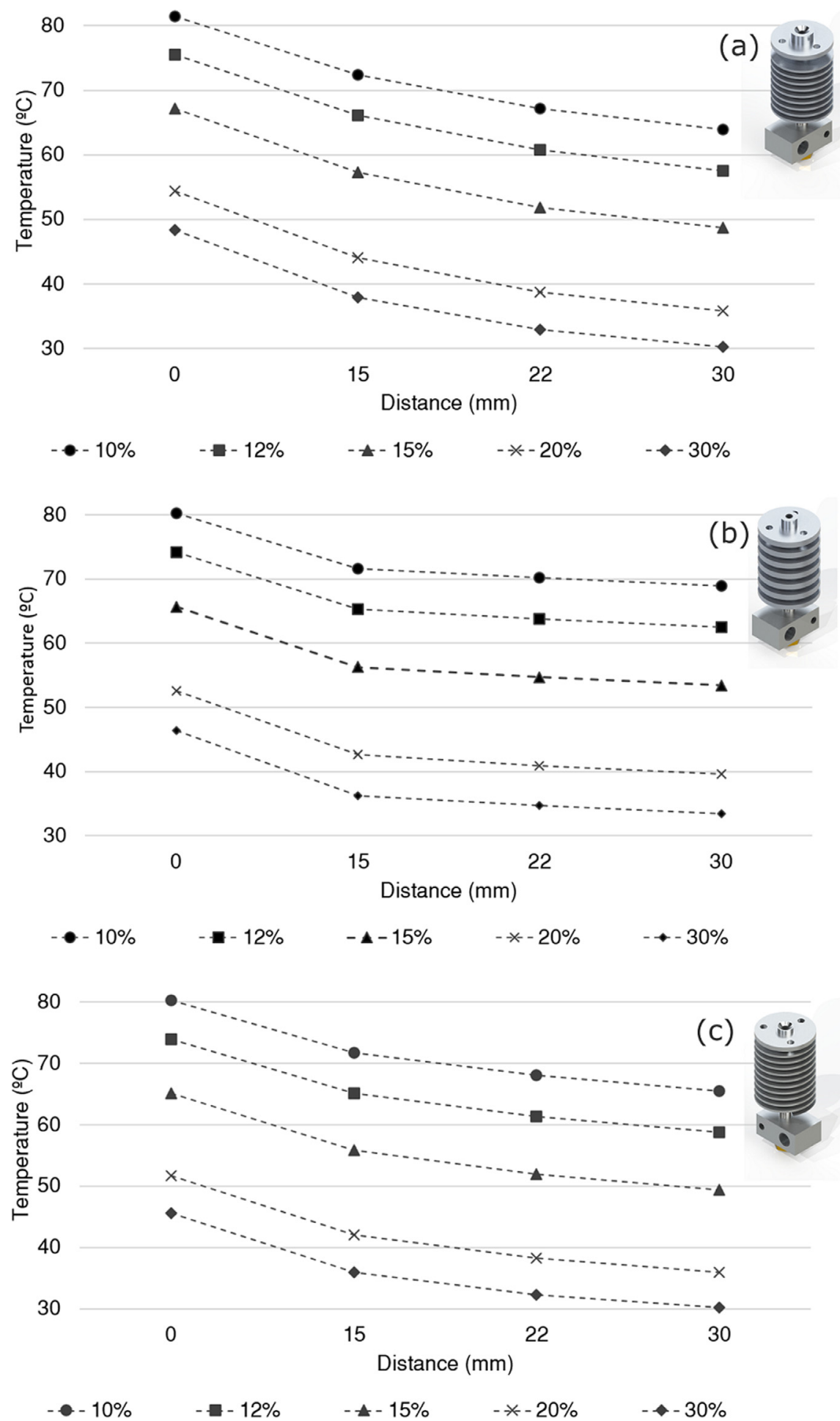

Fig. 6. Temperature profile derived from FEM analysis for the three analyzed geometries. (a) BCNozzle. (b) X-Truder. (c) Twist3D.

ing an objective temperature of $210^{\circ} \mathrm{C}$, characteristic value taken to print of PLA material. Once the thermistor registered for $5 \mathrm{~min}$ a constant temperature around the objective, the four thermocouples recorded the temperature during $2.5 \mathrm{~min}$, at a $62.5-\mathrm{Hz}$ sampling rate, using a TC-08 Thermocouple Data Logger. The resistance was then turned off until it reached ambient temperature. Then, it was once again connected to perform a second measurement, following the same procedure. This cool-down/heat-up cycle, and ulterior measurement, was repeated 12 times for each sink geometry and fan speed, to confirm the repeatability of results. In total, 216 sets of values were recorded. The mean temperature for each set of values was calculated by averaging the registered values, discarding outliers by applying the Chauvenet's criterion.

\section{Results discussion}

\subsection{FEM calculations}

Fig. 6 shows the temperature profiles after the FEM analysis results. They have been represented as a discontinuous linear graph 

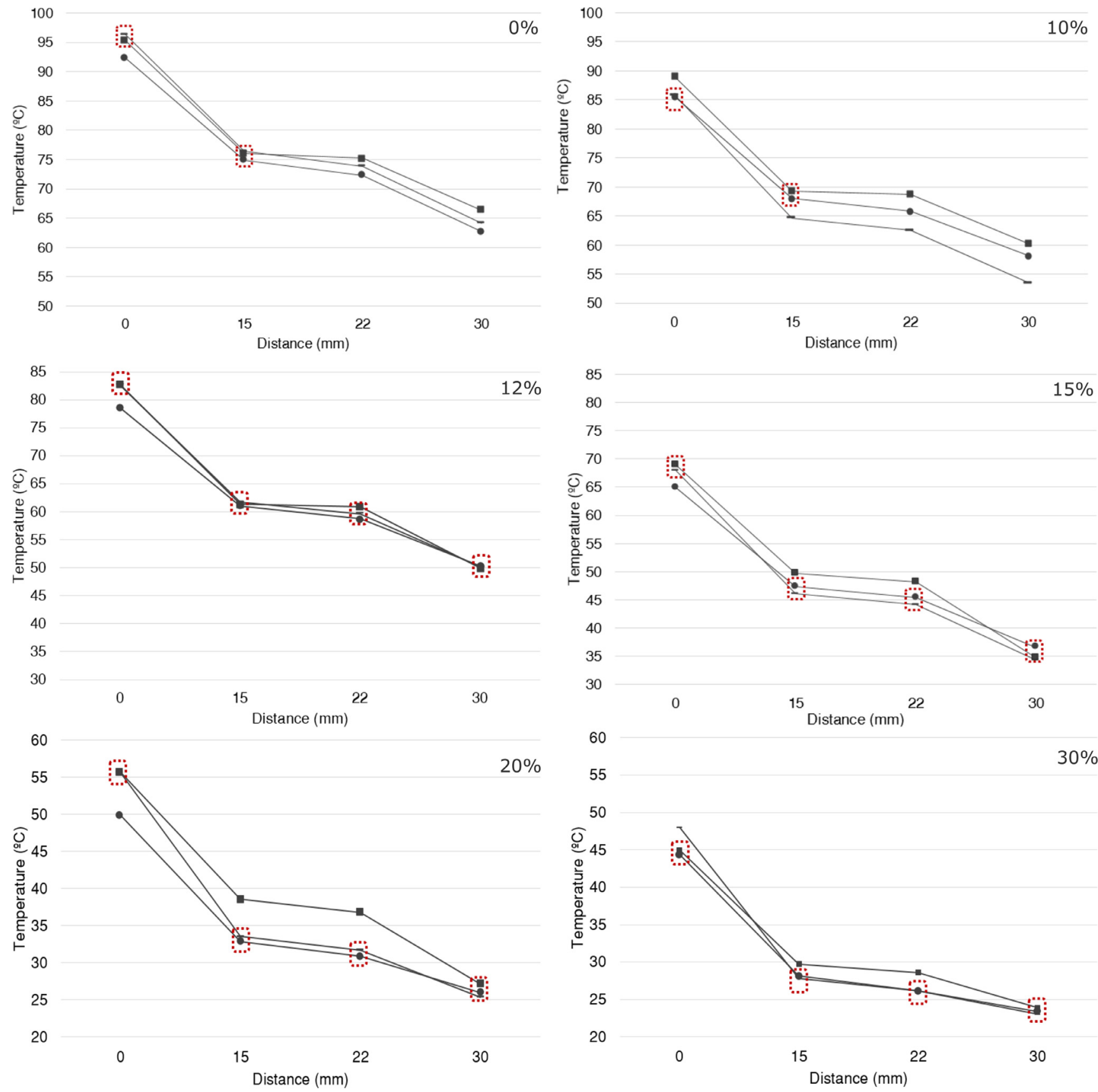

\section{Twist3D \\ - BCNozzle \\ - - X-Truder}

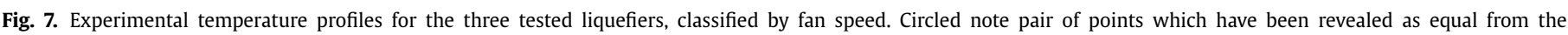
Tukey analysis.

defined by the four measured points. The three liquefier geometries show similar temperature profiles, being the X-Truder liquefier the one which derives in the lowest temperatures at the top of the heat sink $(30 \mathrm{~mm})$. More specifically, according to the model calculations, the difference with regards to the original geometry is about $4^{\circ} \mathrm{C}$. The most remarkable temperature reduction in all geometries can be noticed from 0 to $15 \mathrm{~mm}$ (that is, at the liquefier section next to the heat barrier), but that temperature fall is more pronounced in the X-Truder liquefier. According to these results, it seems that the decision of increasing the fins thickness in this new liquefier design proves to be theoretically valid.

The fan speed shows a more influential effect on temperature reduction at the intermediate tested fan speeds, which means that its behavior is not linear. In effect, the highest temperature reduction is observed when the fan speed is increased from $15 \%$ to $20 \%$, which is much more pronounced than from $20 \%$ to $30 \%$.

\subsection{Experimental results}

The 15 combinations of coupled liquefier geometry and fan speed were implemented and measured during the experimental phase, registering the real temperature profiles at all tested conditions. The temperature profiles with no convective cooling system (fan speed $0 \%$ ) was also recorded as a reference value. Fig. 7 shows the temperature profiles for each fan speed level. The represented points are the average values obtained from each sample. As some 

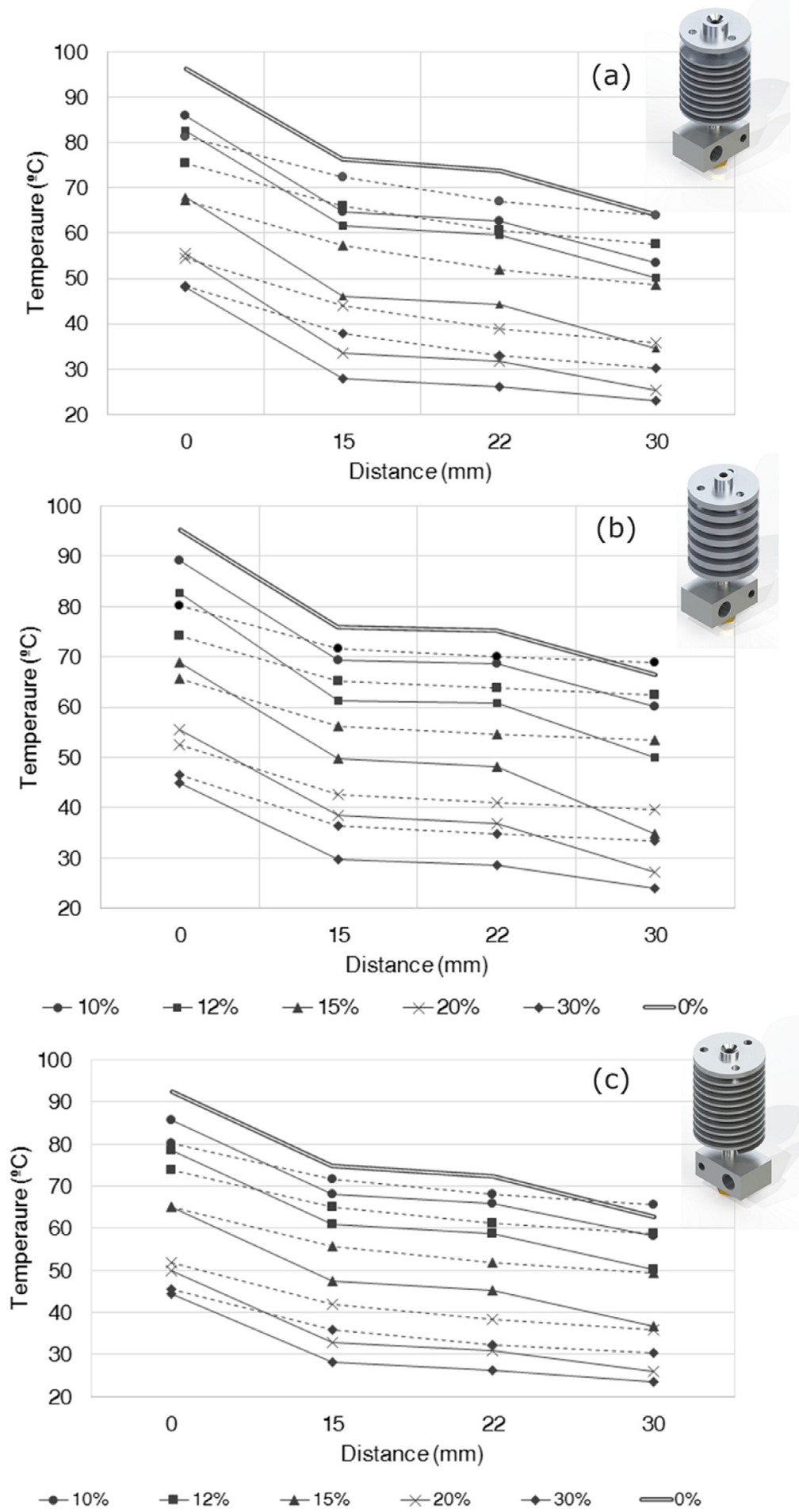

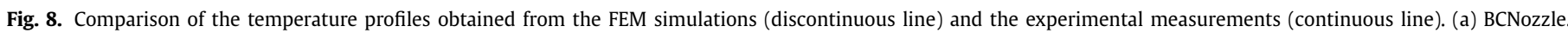
(b) X-Truder. (c) Twist3D.

of them are overlapped, an analysis of variance (ANOVA) was performed with the Minitab 17 software to decide with statistical criteria whether separated points were actually different responses, taking into account the dispersion of the samples. A 95\% confidence level was considered. The ANOVA results were used to apply the Tukey method, which allows to test the equality of temperatures by pairs. Table 2 exemplifies the results of that method for the results corresponding to $10 \%$, at the four monitored liquefier heights. Samples assigned with the same letter can be considered
Table 2

Example of the Tukey statistical comparative method for fan speed $10 \%$. Measures coded with the same letter at each level (shadowed cells) can be considered statistically equal.

\begin{tabular}{lllll}
\hline Liquefier & $0 \mathrm{~mm}$ & $15 \mathrm{~mm}$ & $22 \mathrm{~mm}$ & $30 \mathrm{~mm}$ \\
\hline Twist3D & B & C & B & A \\
BCNozzle & B & B & C & B \\
X-Truder & A & A & A & A \\
\hline
\end{tabular}


equal, and are highlighted in Fig. 7 with a discontinuous circular line.

Results show that there is a different relative behavior of all three liquefier geometries depending on the level of fan speed. For the $0 \%$ and $10 \%$ fan speed cases, the three liquefiers show highly differential temperature profiles, separated by around $3^{\circ} \mathrm{C}$. The $\mathrm{X}-$ Truder reveals to lead to the highest temperatures, followed by the Twist3D and the BCNozzle, which reveals to achieve the lowest temperatures at the liquefier body. In overall, the BCNozzle and the Twist3D have a similar heat dissipation dynamics, and are specially advantageous at fan speeds below $10 \%$.

For fan speeds higher than $10 \%$, the temperature profiles overlap, although the X-Truder shows a general trend to lead to the highest temperatures at 0,15 and $22 \mathrm{~mm}$ height. This is especially visible at the $20 \%$ fan speed temperature profiles. However, all three geometries lead to the same temperature at the top of the liquefier $(30 \mathrm{~mm})$, which is proved by the fact that the Tukey tests show statistical equality of all temperature values for $12 \%$, $15 \%, 20 \%$ and $30 \%$ fan speeds. As this is the critical temperature, the X-Truder proves to be able to reduce the temperature at the top of the heat sink as effectively as the other two designs, while being manufactured through a faster process due to its design simplicity.

\subsection{Comparison between FEM and experimental measures}

Experimental results were represented along with the FEM simulations temperature profiles to compare the behavior of all the liquefiers at all tested fan velocities. Fig. 8 shows this representation for each of the three tested liquefiers, including the temperature profile at the case that no convective heat dissipation is used.

In general terms, the FEM temperature profiles are higher than the actual values measured during the experimental phase. This observation can be explained by the fact that the liquefier is fixed to the support structure through elements which are conductive themselves, and that might lead to heat leaks not represented at the model. However, this fact does not affect the comparison among liquefier results, as it is a constant situation at all experimental setups. On the other hand, the model is based on the fact that temperature at the heat block remains at the same level during the printing process, whereas the feedback loop responsible for temperature regulation leads to an non-constant temperature in the real case.

As the experimental setup is not isolated inside a controlled atmosphere, fluctuations of temperature due to external sources are not included in the analysis. This might be a source of divergence on results, although it must not be forgotten that RepRap 3D printers actually work in open atmospheres, so that no temperature control is performed. These considerations lead to think that the model should be in next steps complemented with more realistic conditions, by reducing simplifications to optimize its calculations.

\subsection{Fan usage and energy consumption}

Results at Fig. 8 show that the effect of the fan speed on the final temperature profile is not linear. This is proved in both the experimental and the Fem results. For instance, for the Twist3D liquefier, the difference between the $10 \%$ and the $20 \%$ temperature profiles is about $35^{\circ} \mathrm{C}$, whereas the difference between the $20 \%$ and the $30 \%$ lines is less than $10^{\circ} \mathrm{C}$. This fact leads to the conclusion that refrigerating with fan speeds higher than $20 \%$ is not advisable, as increasing the velocity to a $30 \%$ level has a feeble effect on final temperature.

To support this hypothesis, the electrical power consumed by the fan for each velocity level was calculated by measuring the demanded voltage and intensity at all PWM percentages levels
Table 3

Electrical power consumed by the cooling fan for every tested airflow.

\begin{tabular}{llll}
\hline PWM fan speed & Voltage $(\mathrm{V})$ & Intensity $(\mathrm{A})$ & Power $(\mathrm{W})$ \\
\hline $10 \%$ & 1.6 & 0.011 & 0.01760 \\
$12 \%$ & 1.8 & 0.0132 & 0.02376 \\
$15 \%$ & 2.1 & 0.0165 & 0.03465 \\
$20 \%$ & 2.75 & 0.022 & 0.06050 \\
$30 \%$ & 4 & 0.033 & 0.13200 \\
\hline
\end{tabular}

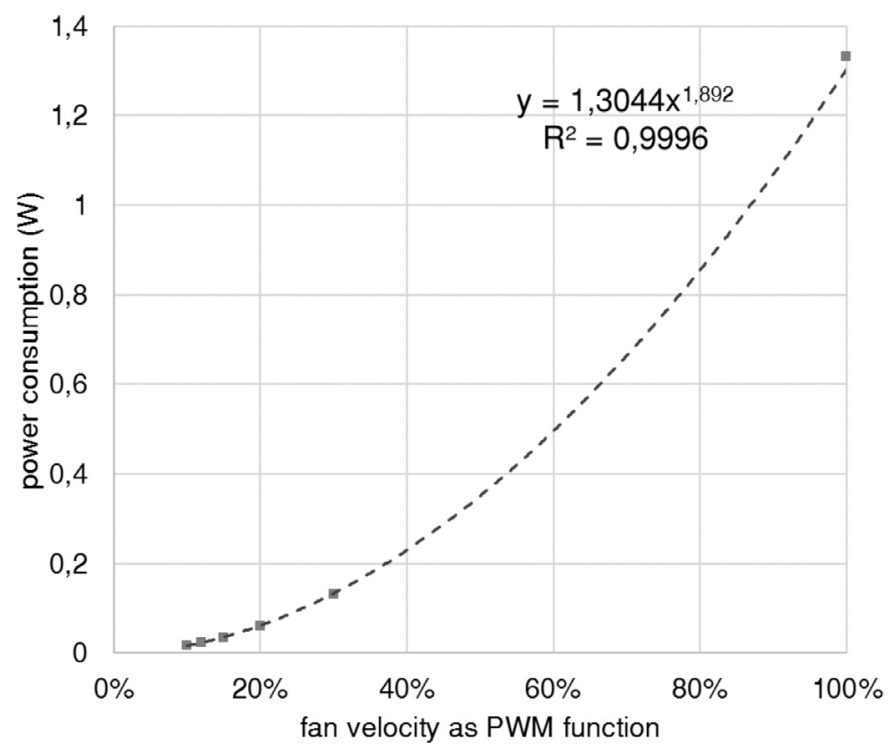

Fig. 9. Exponential relation between PWM fan speed regulation function and power demanded by the fan.

(Table 3). The increase of demanded power by the fan when increasing its PWM fan speed from $10 \%$ to $20 \%$ is of $0.0429 \mathrm{~W}$, whereas the increase from $20 \%$ to $30 \%$, the increase is $0.0715 \mathrm{~W}$. This shows a non-linear relation between fan speed defined as the PWM function, and demanded power. In effect, results can be adjusted to an exponential graph with a correlation ratio of $99.96 \%$ (Fig. 9). As a consequence, increasing the fan speed from $20 \%$ to $30 \%$ is strongly unadvised, as the fan consumption is higher, whereas the actual effect is not remarkable.

\section{Conclusions}

The temperature profiles of three different 3D printer liquefier have been analyzed through a FEM simulation and experimental measurements. The results observed and presented at this paper lead to the following conclusions:

1. The whole temperature profiles for the different measured cases show that the Twist3D design presents slightly higher temperatures at the intermediate points of the liquefier. However, when a fan speed higher than $10 \%$ is used, it succeeds in achieving the same temperature at the top of the nozzle. Therefore, the Twist3D liquefier proves to be an improved design of the heat sink geometry, provided the higher simplicity in terms of manufacturing complexity.

2. For the three tested geometries, it is strongly advised to refrigerate the printing system using a $20 \%$ fan speed of the PWM function. This is due to a double reason. First of all, that temperature reduction has not a liner relation with fan speed, and is considerably lower when increasing the PWM value from $20 \%$ to $30 \%$. Secondly, that same increase requires nonetheless a higher power consumption, if compared with increasing from 
$10 \%$ to $20 \%$, so that the efficiency of a higher fan speed decreases at levels higher than $20 \%$.

3. The finite elements model should be tuned to reach results nearer to the experimental measured values, as FEM results are higher at all tested conditions. This could be due to the fact that the real thermal inflow is performed by a resistance which is turned on and off alternatively by means of a simple feedback system, which can affect results. This improvement is necessary if the model is to be applied to further thermal behavior studies, such as characterization of the melt front inside the liquefier or improving hardware defects such as nozzle blocking for inadequate temperature.

\section{References}

[1] Wittbrodt BT, Glover AG, Laureto J, Anzalone GC, Oppliger D, Irwin JL, et al. Life-cycle economic analysis of distributed manufacturing with opensource 3-D printers. Mechatronics 2013;23(6):713-26.

[2] RepRap Project Wiki. www.reprap.org. Last retrieved on 12/11/2016,

[3] Kulkarni P, Marsan A, Dutta D. A review of process planning techniques in layered manufacturing. Rapid Prototyping J 2000;6(1):18-35.

[4] Lanzotti A, Del Giudice DM, Lepore A, Staiano G, Martorelli M. On the geometric accuracy of RepRap open-source three-dimensional printer. J Mech Des 2015;137(10):1017031-8.

[5] Turner N, Strong B, Gold SA. A review of melt extrusion additive manufacturing processes: I. Process design and modeling. Rapid Prototyping J 2014:20(3):192-204.

[6] Domingo-Espin M, Borros S, Agullo N, Garcia-Granada AA, Reyes G. Influence of building parameters on the dynamic mechanical properties of polycarbonate fused deposition modeling parts. 3D Print Addit Manuf 2014;1(2):70-7.

[7] Bellehumeur C, Li L, Sun Q Gu P. Modeling of bond formation between polymer filaments in the fused deposition modeling process. J Manuf Process 2004;6(2):170-8.

[8] Bellini A, Güçeri S. Mechanical characterization of parts fabricated using fused deposition modeling. Rapid Prototyping J 2003;9(4):252-64.

[9] Sun Q Rizvi GM, Bellehumeur CT, Gu P. Effect of processing conditions on the bonding quality of FDM polymer filaments. Rapid Prototyping J 2008;14(2):72-80

[10] Domingo-Espin M, Puigoriol-Forcada JM, Garcia-Granada AA, Llumà J, Borros S, Reyes G. Mechanical property characterization and simulation of fused deposition modeling polycarbonate parts. Mater Des 2015;83:670-7.
[11] Zhang Y, Chou K. A parametric study of part distortions in fused deposition modelling using three-dimensional finite element analysis. P I Mech Eng B-J Eng 2008;222(8):959-68.

[12] Nickel AH, Barnett DM, Prinz FB. Thermal stresses and deposition patterns in layered manufacturing. Mater Sci Eng A-Struct 2001;317(1):59-64.

[13] Zhang Y, Chou YK. Three-dimensional finite element analysis simulations of the fused deposition modelling process. P I Mech Eng B-J Eeng 2006;220(10):1663-71.

[14] Huang HX, Peng YC. Theoretical modeling of dispersive melting mechanism of Polymers in an Extruder. Adv Polym Tech 1993;12(4):343-52.

[15] Yarimci MA. Conceptual framework for the thermal process modelling of fused deposition. Prot J 1996;2(2):26-31.

[16] Yarimci MA, Hattori T, Guceri I, Danforth SC. Thermal analysis of fused deposition. In: Proceedings of solid freeform fabrication conference; 1997. p. 689-98.

[17] Bellini A, Guçeri S, Bertoldi M. Liquefier dynamics in fused deposition. J Manuf Sci E-T ASME 2004;126(2):237-46.

[18] Bellehumeur C, Li L, Sun Q, Gu P. Modeling of bond formation between polymer filaments in the fused deposition modeling process. J Manuf Proc 2004;6(2):170-8.

[19] Ramanath HS, Chua CK, Leong KF, Shah KD. Melt flow behaviour of poly- $\varepsilon$-caprolactone in fused deposition modelling. J Mater Sci-Mater M 2008;19(7):2541-50.

[20] Nikzad M, Syed HM, Igor S, Andrew G. A study of melt flow analysis of an ABS-Iron composite in fused deposition modelling process. Tsinghua Sci Tech 2009;14:29-37.

[21] Nikzad M, Masood SH, Sbarski I. Thermo-mechanical properties of a highly filled polymeric composites for fused deposition modeling. Mater Design 2011:32(6):3448-56.

[22] Monzon MD, Gibson I, Benítez AN, Lorenzo L, Hernández PM, Marrero MD. Process and material behavior modeling for a new design of micro-additive fused deposition. Int Journal Adv Manuf Tech 2013;67(9-12):2717-26.

[23] Jerez-Mesa R, Travieso-Rodriguez JA, Corbella X, Busque R, Gomez-Gras G. Finite element analysis of the thermal behavior of a RepRap 3D printer liquefier. Mechatronics 2016;36:119-26.

[24] Bassam AH. Fin placement for optimal forced convection heat transfer from a cylinder in cross-flow. Int J Num Meth Heat Fluid Flow 2005;15(3):277-95.

[25] Barbu V. Stabilization of Navier-Stokes flows. 1st ed. London: Springer; 2011.

[26] Reddy JN, Gartling DK. The finite element method in heat transfer and fluid dynamics. 3rd ed. New York: CRC Press, Taylor \& Francis Group; 2010.

Ramón Jerez Mesa is Industrial Engineer and PhD Candidate at the Mechanical Engineering Department of the Polytechnic University of Catalonia. His research activity focuses on ultrasonic finishing processes and the characterization of rapid manufacturing and 3D printing with FFF. 